\title{
Identification of novel biomarkers for neonatal hypoxic-ischemic encephalopathy using iTRAQ
}

Yuanyuan Zhu ${ }^{1 \dagger}$, Yajing Yun ${ }^{1 \dagger}$, Meifang $\mathrm{Jin}^{2}$, Gen Li ${ }^{2}$, Hong Li', Po Miao ${ }^{1}$, Xin Ding ${ }^{1}$, Xing Feng ${ }^{1}$, Lixiao Xu ${ }^{2^{*}}$ and $\operatorname{Bin} \operatorname{Sun}^{1 *}(1)$

\begin{abstract}
Background: A prompt diagnosis of HIE remains a challenge clinically. This study aimed to identify potential biomarkers of neonatal hypoxic-ischemic encephalopathy (HIE) via a novel proteomic approach, the isobaric tags for absolute and relative quantification (iTRAQ) method.

Methods: Blood samples were collected from neonates with mild $(n=4)$, moderate $(n=4)$, or severe $(n=4)$ HIE who were admitted to the neonatal intensive care unit of Children's Hospital of Soochow University between Oct 2015 and Oct 2017. iTRAQ was performed in HIE patients and healthy controls $(n=4)$. Bioinformatics analyses including Gene Ontology and KEGG pathway enrichment analysis were performed to evaluate the potential features and capabilities of the identified differentially expressed proteins.
\end{abstract}

Results: A total of 51 commonly differentially expressed proteins were identified among the comparisons between mild, moderate, and severe HIE as well as healthy controls. Haptoglobin (HP) and S100A8 were most significantly up-regulated in patients with HIE and further validated via real-time PCR and western blotting. The differentially expressed proteins represented multiple biological processes, cellular components and molecular functions and were markedly enriched in complement and coagulation cascades.

Conclusions: HP and S100A8 may serve as a potential biomarker for neonatal HIE and reflects the severity of HIE. The complement and coagulation cascades play crucial roles in the development of neonatal HIE.

Keywords: Neonate, Hypoxic-ischemic encephalopathy, Isobaric tags for absolute and relative quantification, Haptoglobin, S100A8

\section{Introduction}

Neonatal hypoxic ischemic encephalopathy (HIE) is a severe form of neonatal brain damage caused by an asphyxia-related decrease in cerebral blood flow and hypoxia. It is estimated that 4 million out of approximately 130 million newborns suffer from asphyxia

\footnotetext{
*Correspondence: xulixiao2013@hotmail.com; sunyu0628@126.com †Yuanyuan Zhu and Yajing Yun contributed equally to this work. ${ }^{2}$ Institute of Pediatric Research, Children's Hospital of Soochow University, Suzhou, China

'Department of Neonatology, Children's Hospital of Soochow University, Suzhou, China
}

worldwide per year, of which 1 million die and 1 million develop serious and long-term sequelae [1]. In China, the incidence of newborn HIE is about 54,000-108,000 in up to 20 million live births per year, of which 8000 20,000 die in the neonatal period, and $25-30 \%$ of the survivors are affected by long-term sequelae such as cerebral palsy, epilepsy, and mental retardation [2].

An early diagnosis of HIE is essential for subsequent treatment and prognosis. However, prompt diagnosis of HIE remains a challenge clinically partly due to its unclear pathogenesis and rapid progression. Previous

(c) The Author(s). 2020 Open Access This article is licensed under a Creative Commons Attribution 4.0 International License, which permits use, sharing, adaptation, distribution and reproduction in any medium or format, as long as you give appropriate credit to the original author(s) and the source, provide a link to the Creative Commons licence, and indicate if changes were made. The images or other third party material in this article are included in the article's Creative Commons licence, unless indicated otherwise in a credit line to the material. If material is not included in the article's Creative Commons licence and your intended use is not permitted by statutory regulation or exceeds the permitted use, you will need to obtain permission directly from the copyright holder. To view a copy of this licence, visit http://creativecommons.org/licenses/by/4.0/ The Creative Commons Public Domain Dedication waiver (http://creativecommons.org/publicdomain/zero/1.0/) applies to the data made available in this article, unless otherwise stated in a credit line to the data. 
studies revealed that biomarkers such as brain-specific proteins (neuron-specific enolase (NSE), S100B, ubiquitin carboxy-terminal hydrolase-L1, total Tau) and cytokines, including interleukin (IL)-6, IL-1 $\beta$, IL-10, IL-13, interferon-gamma, tumor necrosis factor alpha and brain-derived neurotrophic factor, are helpful in the diagnosis of HIE and prediction of neurodevelopment outcomes [3-6]. However, optimal sensitivity and specificity of these biomarkers have not been achieved, which has limited their clinical application [5].

Advances in proteomics technology have brought new hope for the identification of novel biomarkers for a number of diseases. As a critical component of postgenomic research, proteomics includes two research strategies, namely "complete proteomics" and "differential proteomics". Differential proteomics is mainly applied to screening for differences in proteomes between different samples. The proteomics diversity further reveals the physiological and pathological status of cells, the underlying mechanisms of cell activity, and the features of involved key proteins [7]. To date, to our knowledge, there has been no study evaluating biomarkers of neonatal HIE using proteomics technology.

In the current study, the similarities and differences in serum protein expression between newborn HIE patients and healthy newborns were detected by a novel isobaric tag for absolute and relative quantitation (iTRAQ)-based quantitative clinical proteomics approach. We attempted to identify novel biomarkers for neonatal HIE. Our results not only provide a theoretical basis for uncovering the disease pathogenesis of neonatal HIE, but also may serve as a scientific basis for the development of further treatment strategies.

\section{Methods}

\section{Study cohort}

In this prospective study, full-term newborn infants diagnosed with HIE and admitted to the neonatal intensive care unit of Soochow University Children's Hospital between October 2015 and October 2017 were enrolled as the HIE group. The diagnosis of HIE was based on the modified 2004 HIE diagnostic and indexing criteria of the Neonatology Group of the Pediatric Society of the Chinese Medical Association [8]. All HIE patients were evaluated within the first 3 days after birth. Healthy fullterm neonates born at the municipal hospital of Suzhou during the same period whose characteristics including gender, birth weight, and delivery patterns were well matched with the HIE group were selected as the controls. Patients with severe infection, malformation, bilirubin encephalopathy or incomplete data were excluded from the HIE group. Patients were excluded from the control group if they had a history of asphyxia or if the pregnant mother had underlying diseases. A total of 12
HIE patients (4 for each HIE group, mild HIE, moderate HIE and severe HIE) and 4 healthy controls were selected for iTRAQ analysis. These HIE patients were randomly selected from the 57 initially enrolled patients, which included 27 cases of mild HIE, 16 of moderate HIE, and 14 of severe HIE. Sixteen healthy patients were included as the controls.

\section{Data collection}

Characteristics including gender, gestational age, birth weight, delivery mode, and length of hospital stay were recorded. General conditions including consciousness, muscle tone, reflexes, convulsions, respiratory failure, and pupillary changes were evaluated. Laboratory measurements and radiological imaging were performed.

\section{Sample preparation}

All patients and healthy controls were at approximately the same age (37-38 weeks). Blood samples $(3 \mathrm{ml})$ were collected before the initiation of treatment after birth. For healthy controls, identical volumes of blood samples were collected. Blood samples were not collected during therapeutic hypothermia. All blood samples were collected in EDTA tubes, and plasma was obtained by centrifugation at $3000 \mathrm{~g}$ for $10 \mathrm{~min}$. Aliquots of plasma were taken and stored at $-80^{\circ} \mathrm{C}$. Immunoglobulin (IgG) and albumin were removed from the samples $(40 \mu \mathrm{l})$ using the Qproteome Albumin/IgG Depletion Kit (Qiagen, Valencia, CA, USA). After depletion of these high-abundance proteins, the samples were centrifuged at $12000 \mathrm{~g}$. The protein content of each sample was determined using the Pierce BCA Protein Assay (Thermo Fisher Scientific, Rockford, IL, USA). Proteins $(15 \mu \mathrm{g})$ from each sample were separated by sodium dodecyl sulfate (SDS)-polyacrylamide gel electrophoresis (PAGE) on $12 \%$ polyacrylamide gels and stained with Coomassie blue. The stained gel was scanned by the Image Scanner (GE Healthcare, USA) at a resolution of 300 dots per inch.

Extracted proteins $(100 \mu \mathrm{g})$ were digested with trypsin at $37^{\circ} \mathrm{C}$. Peptides were eluted from filters with $100 \mathrm{mM}$ ammonium bicarbonate buffer and purified. iTRAQ labeling was subsequently performed using an 8-plex iTRAQ labeling kit (AB Sciex, USA; catalogue: 4381664) according to the manufacturer's protocol. Controls were labeled with iTRAQ reagents 113 and 114; mild HIE samples with iTRAQ reagents 115 and 116; moderate HIE samples with 117 and 118; severe HIE samples with 119 and 121. The labeled digest samples from the four groups were combined into one tube.

\section{LC-MS/MS analysis}

An EASY-nLCTM 1200 UHPLC system (Thermo Fisher, Waltham, MA, USA) was coupled to a Orbitrap Q Exactive HF-X mass spectrometer (Thermo Fisher). Columns 
packed with ChromXP C18 $(100 \mu \mathrm{m} \times 3 \mathrm{~cm}, 3 \mu \mathrm{m}, 150$ $\AA$, Eksignet) were used for online trapping and desalting, while columns packed with Chrom XP C18 $(75 \mu \mathrm{m} \times 15$ $\mathrm{cm}, \mathrm{C} 18,3 \mu \mathrm{m}, 120 \AA$, Eksigent) were utilized for analytical separation. The mobile phase A was composed of acetonitrile/water/formic acid (ACN-H2O-FA 2: 98: 0.1, $\mathrm{v} / \mathrm{v} / \mathrm{v}$ ), and the mobile phase B of ACN-H2O-FA (95: 5: $0.1, \mathrm{v} / \mathrm{v} / \mathrm{v})$. Samples were loaded on the column with trapping and desalting carried out at $3 \mu \mathrm{L} / \mathrm{min}$ for 10 min using mobile phase A. Analytical separation was performed at a flow rate of $300 \mathrm{~nL} / \mathrm{min}$. Key parameter settings included first grade MS parameters of a resolution of 70,000, Automatic Gain Control (AGC) target of $1 \mathrm{e} 6$, and scan range of $300 \sim 1800 \mathrm{~m} / \mathrm{z}$; and second grade MS parameters of resolution of 35,000, AGC target of 5e5, maximum Ion Accumulation Time (IT) of $100 \mathrm{~ms}$, preferred dynamic exclusion time of 30s, and Normalized Collision Energy (NCE)/stepped NCE of 28.

\section{Database search and analysis of differentially expressed proteins}

Protein identification was performed using the Proteome Discoverer $^{\text {rn }}$ software (version 1.3, Thermo Scientific). The RAW files were converted to a ProteinPilot compatible Mascot Generic Format (MGF) with pre-selected iTRAQ reporter ions. The files were searched against the Uniprot Homo sapiens database with the following search parameters: sample type, iTRAQ 8-plex (Peptide Labeled); Cysalkylation, iodoacetamide; digestion, trypsin; instrument, Q Exactive; database, Homo sapiens; and Fasta.

Proteins identified at a $1 \%$ false discovery rate (FDR) with a confidence threshold of 95\% (Proteinpilot Unused score $\geq 1.2$ ) in all runs and $p$ values less than 0.05 in at least one pairwise comparison were retained.

\section{Bioinformatics analyses of differentially expressed proteins}

The functional analysis was performed through the OmicsBean cancer data analysis platform. The linked databases were Quick GO (https://www.ebi.ac.uk/ QuickGO/) and Kyoto Encyclopedia of Genes and Genomes (KEGG) pathway (http://www.kegg.jp/kegg/patyway.html). Gene ontology (GO) analysis included biological processes, cellular components and molecular functions. KEGG pathway analysis was conducted to identify pathway clusters. A $p$ value less than 0.05 was set as the cutoff criterion.

\section{Polymerase chain reaction (PCR) analysis and western blot analysis for validation of biomarkers}

Total RNA from the samples was isolated in two steps using Trizol reagent (Invitrogen, Carlsbad, CA, USA) followed by RNeasy (Qiagen) purification and then subjected to reverse transcription. Haptoglobin (HP),
S100A8, apolipoprotein E (APOE), and apolipoprotein $M$ (APOM) expression levels were amplified with primers (5'-TAGAGACCGAGTGTCCTCA-3', 5' CGCCCATCTTTATCACCAGA-3', 5' -CAGCACAGTC CCCGAAAAGAA-3', 5' - CAGTCGCATACCAGGTGT CC-3', 5' -TGACGCTGGGGCTGGCATTG-3', 5' GCTCTTGCTGGGGCTGGTGG-3', $5^{\prime}$ - $\quad$ GTTGCT GGTCACATTCCTGG-3', 5' - GCAGGTAATCCCAA AAGCGAC-3', 5' - GCTACCATCCGCATGAAAGAT3', 5'- CTGGCCTGTCTCATTCAGCA-3'). The PCR reactions were performed in a $10 \mu \mathrm{L}$ volume containing a $2 \times$ SYBR Green Master Mix and the reaction was carried out using a fluorescent quantitative real-time PCR (Roche LightCycler 480, Roche, Switzerland). The amplification parameters were $95^{\circ} \mathrm{C}$ for $5 \mathrm{~min}$; followed by 45 cycles of $95^{\circ} \mathrm{C}$ for $1 \mathrm{~min}$ and, $60^{\circ} \mathrm{C}$ for $20 \mathrm{~s}$, and $72{ }^{\circ} \mathrm{C}$ for $20 \mathrm{~s}$. $\beta$-actin was used as an internal control to ensure cDNA quality and loading accuracy. For every transcript, each cDNA sample was analyzed in triplicate. The assessment of expression comparing different targets was determined by the ddCt comparative threshold $\left({ }^{\Delta \Delta} \mathrm{Ct}\right)$ method. P-values were determined by a twotailed paired Student's t-test.

Plasma proteins were separated by SDS-PAGE and transferred to polyvinylidene fluoride (PVDF) membranes. The membranes were blocked with blocking reagent and then incubated with primary antibody at a concentration of 1: 1000 (Santa Cruz Biotechnology, Santa Cruz, CA, USA), overnight at $4{ }^{\circ} \mathrm{C}$, followed by secondary antibody at a concentration of 1:5000 (Santa Cruz Biotechnology) for $1 \mathrm{~h}$ at room temperature. A Western Imaging System (Thermo Fisher Scientific) was used to obtain the images and analyze target protein expression.

\section{Statistical analysis}

Statistical analysis was carried out using SPSS Statistics 22.0 (IBM, Armonk, NY, USA). Graphs were prepared using GraphPad Prism 7 software (GraphPad, Inc., San Diego). Variables were described as mean \pm standard deviation or median (interquartile) as appropriate. Continuous variables were compared with a t-test or MannWhitney U test, whereas categorical data were compared with chi-square test or Fisher's exact test. Differences in protein expression between groups were analyzed using a t-test. Values of p less than 0.05 were considered statistically significant.

\section{Results}

Baseline characteristics of HIE patients and healthy controls

Of the 12 cases of HIE for which iTRAQ analysis was performed, male and female patients accounted for 7 (58.3\%) and 5 (41.7\%) cases, respectively. Blood samples were withdrawn before the initiation of treatment within 
a mean time of $5.19 \pm 2.12 \mathrm{~h}$ after birth. For healthy controls, identical volumes of blood samples were withdrawn at a mean time of $6.46 \pm 1.79 \mathrm{~h}$ after birth. There were no significant differences in gender and time before sampling between the HIE and control groups (both $p>$ 0.05).

\section{Differentially expressed proteins identified by ITRAQ analysis}

From the comparison of mild HIE cases versus controls, 69 differentially distributed proteins were detected, including 18 up-regulated proteins and 51 down-regulated proteins. The significantly differentially expressed proteins were illustrated in a volcano plot, and the expression levels of proteins in each sample were visualized in a hierarchical clustering heatmap (Fig. 1a, b). From the comparison of moderate HIE cases versus controls, 115 differentially expressed proteins were detected, of which 27 were up-regulated and 88 were down-regulated (Fig. 1c, d). From the comparison of severe HIE cases versus controls, 133 differentially expressed proteins were identified, including 14 up-regulated proteins and 119 downregulated proteins (Fig. 1e, f).

To identify commonly differentially expressed proteins within the above three comparisons, a Venn diagram was drawn and showed that 51 proteins were identified in multiple comparisons (Fig. 2, Table 1).

\section{Bioinformatics analyses of differentially expressed proteins}

GO analysis explored the biological processes (BPs), cell components (CC) and molecular functions (MF) associated with the top 20 most positively and negatively regulated proteins. Comparing mild HIE cases with controls, the BP involvement of the most differentially expressed proteins included the injury response, acute inflammatory response, and inflammatory response. Involved CCs were extracellular space, extracellular membrane organelles, and exosomes. Participating MFs included peptidase regulation agent, heparin binding, and glycosaminoglycan binding. KEGG analysis showed that differentially expression proteins were markedly enriched in complement and coagulation cascades. The proteins most differentiated between moderate HIE cases and controls were associated with BPs, CCs and MFs similar to those of the proteins differentially expressed between mild HIE cases and controls. Slight differences in the BPs, CCs and MFs were detected (Fig. 3).

\section{Validation of the identified potential biomarkers}

Eight healthy newborns, 19 patients with mild HIE, 8 with moderate HIE, and 6 with severe HIE were chosen for further validation of the iTRAQ findings. The PCR results showed that, consistent with the findings of
iTRAQ, HP and S100A8 were up-regulated in patients with HIE. However, conflicting results for the proteins APOE and APOM were observed. Western blot analysis ( $n=4$ for each group) further verified the increased expression of HP and S100A8 in patients with HIE (Fig. 4).

\section{Discussion}

The current study investigated diagnostic biomarkers of neonatal HIE by screening differentially expressed proteins between HIE patients and healthy controls. To the best of our knowledge, this study was the first to detect potential biomarkers of neonatal HIE using novel iTRAQ technology. We found that S100A8 may serve as a potential biomarker and HP was closely linked with HIE. Bioinformatics analysis suggested that the complement and coagulation cascades play critical roles in the development of neonatal HIE.

Our results confirmed that S100A8 expression was significantly elevated in all three groups of HIE patients and reflected the severity of HIE. No evidence of the relationship between S110A8 and HIE has been previously reported. However, as an important S100 protein family member, S100A8 is involved in a number of physiological functions including the inflammatory response and calcium homeostasis regulation through combination with S100A9 [9]. The S100A8/A9 complex has been reported to be capable of inhibiting excessive oxidation and facilitating production of pro-inflammatory cytokines $[10,11]$. The S100A8/A9 complex also has been shown to participate in multiple diseases such as asthma and rheumatoid arthritis [12, 13]. For patients with brain injury, the S100A8/A9 complex was shown to be involved in the early stage of cerebral ischemiareperfusion injury [14]. Previous studies demonstrated that S100A8/A9 mediates early inflammatory responses to neuronal damage by activating microglia Toll-like receptor 4 (TLR4)/nuclear factor kappa B (NF-kB) and receptor for advanced glycation endproducts (RAGE)/NF$\mathrm{\kappa B}$ signaling pathways [15]. Therefore, S100A8 may be a potential biomarker for the diagnosis of HIE and also the monitoring of the disease progression.

The HP protein inhibits the hemoglobin-related cerebral inflammatory response, and elevated HP expression may indicate the development of HIE [16]. We observed a consistent increase in HP expression in patients independent of the severity of HIE, and the trend in expression was not parallel with the severity. HP is an acute phase protein that is the most abundant endogenous hemoglobin-binding protein in plasma [17]. Studies have shown that HP is expressed in the brain and has a neuroprotective effect [18]. HP can protect neurons from the cytotoxic effects of hemolysates after intracranial hemorrhage, which is one of the major manifestations of HIE. HP acting as an antioxidant could remove free 


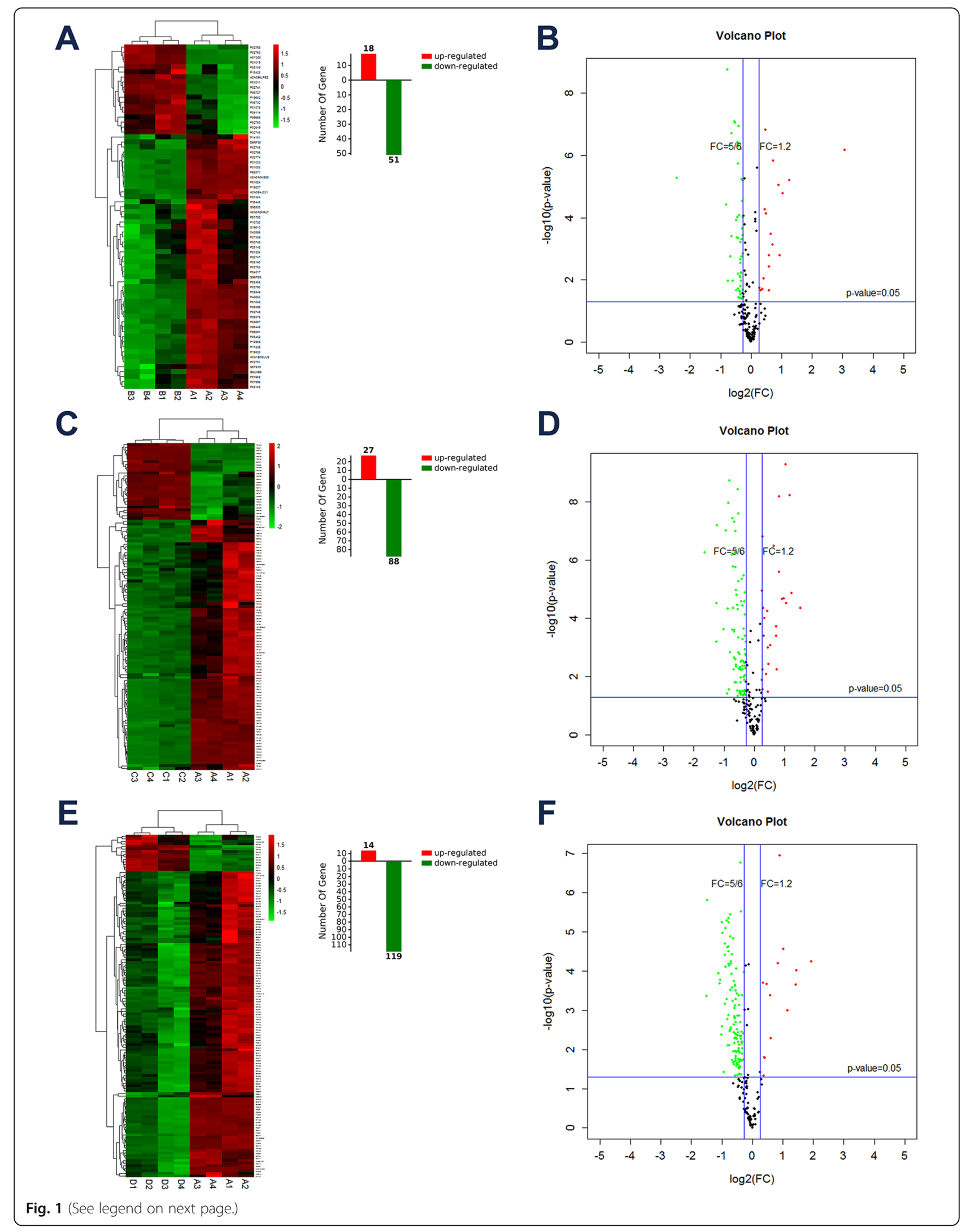


(See figure on previous page.)

Fig. 1 Differentially expressed proteins among HIE patients and healthy controls. a, b Clustering heatmap and Volcano map of proteins differentially expressed between mild HIE and controls. c, d Clustering heatmap and Volcano map for the comparison of moderate HIE vs control. e, $\mathbf{f}$ Clustering heatmap and Volcano map for the comparison of severe HIE vs control. The green dots indicate down-regulated proteins, and the red dots indicate up-regulated proteins. The black dots depict the non-significant differentially expressed proteins

hemoglobin from circulation and protect subsequent impairment due to oxygen free radicals [16]. In patients with severe spinal cord injury, HP expression is increased [19]. Previous in vivo and in vitro studies reported that up-regulation of HP in brain is mainly mediated by oligodendrocytes [20]. However, HP expression has rarely been investigated in newborns previously. Our findings imply that HP could be a definitive marker of HIE, especially mild HIE or early-stage of HIE.

Bioinformatics analysis suggested that the injury response, inflammatory response, and protein activation cascade are important biological processes during the development of HIE. More importantly, KEGG pathway analysis revealed that the complement and coagulation cascades participate the most in all groups. The complement proteins are largely present in human blood and tissue fluid as well as on the cell surface. They function in immunomodulation, immune defense and phagocytosis enhancement [21]. Studies have shown that the blood-brain barrier is impaired via activation of complement proteins, which facilitate histamine and lysosomal

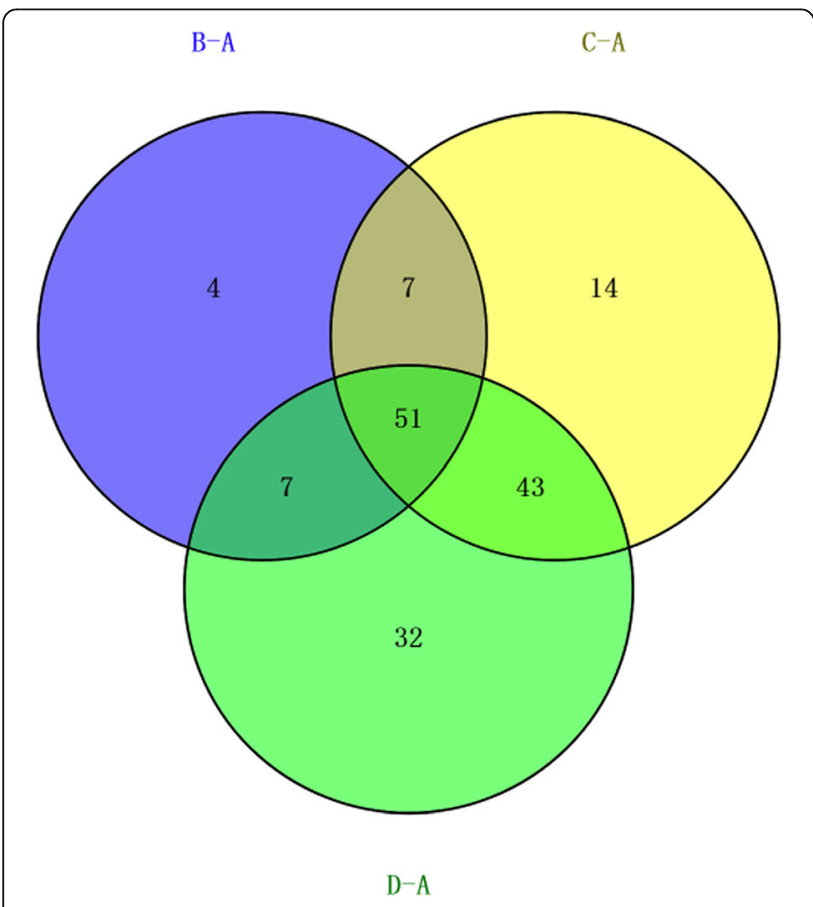

Fig. 2 Venn diagram depicting 51 commonly differentially expressed proteins in all three comparisons of mild HIE vs controls, moderate HIE vs controls, and severe HIE vs controls enzyme release, and increased vascular permeability [22]. Degeneration and necrosis of the brain induce inflammation via activating the complement system [23]. In a rat model of HIE, researchers found that receptors for C3a and C5a were predominantly expressed on microglia after HIE, and hypothermia may improve outcomes by modulating the complement system [24].

Our study based on iTRAQ demonstrated that the coagulation pathway was greatly involved in the development of HIE. Coagulopathy has been proven to be one of the major consequences of neonatal asphyxia [25, 26]. Coagulation disorders following neonatal HIE have multifactorial mechanisms. The synthesis of platelets and clotting factors are inhibited due to insufficient blood and oxygen supply [25-28]. Disseminated intravascular coagulation is frequently present in newborns who suffer from asphyxia [29, 30]. Furthermore, therapeutic hypothermia can potentially disturb hemostasis by slowing the enzymatic function of the coagulation cascade, suppressing thrombin generation, and triggering disseminated intravascular coagulation [31, 32]. Early intervention with coagulation function can improve the prognosis in neonates with HIE. Our results confirmed the concept that the coagulation pathway plays an important role in the progression of HIE.

There are several limitations in the current study. First, the study sample was small due to the limitations of iTRAQ measurement. Second, we did not validate all differentially expressed proteins. However, our findings were partly consistent with the concept that multi-organ and response systems including the oxidative/inflammatory and coagulation cascades are involved in the development of HIE [5]. The differing results among studies may be explained by differences in measurement timing and the related short-or long-term outcomes. In addition, the sensitivity and specificity of different measurements may affect the outcomes as well. Third, we did not analyze the relationship between potential biomarkers and other objective measurements reflecting long-term outcome such as brain MRI. More precise brain imaging and cerebral function assessment may provide further information for the detection of biomarkers. In previous studies assessing the correlations between biomarkers and HIE outcomes, elevated plasma brain-specific proteins including S100B in the first $24 \mathrm{~h}$ after birth were associated with brain injury detected by MRI in HIE newborns [4]. However, S100B was not found to be related to neurodevelopmental outcomes 
Table 1 List of the most differentially expressed proteins in comparisons of mild HIE vs controls, moderate HIE vs controls, and severe HIE vs controls

\begin{tabular}{|c|c|c|c|c|c|c|}
\hline Description & $\begin{array}{l}\text { Mild HIE vs controls } \\
\text { (fold change) }\end{array}$ & $p$ & $\begin{array}{l}\text { Moderate HIE vs controls (fold } \\
\text { change) }\end{array}$ & $p$ & $\begin{array}{l}\text { Severe HIE vs controls } \\
\text { (fold change) }\end{array}$ & $p$ \\
\hline S100A8 & 1.92 & 0.002 & 2.87 & $<0.001$ & 3.85 & $<0.001$ \\
\hline C-reactive protein & 2.38 & $<0.001$ & 2.35 & $<0.001$ & 2.69 & $<0.001$ \\
\hline Apolipoprotein E & 1.21 & 0.019 & 0.73 & 0.003 & 0.72 & 0.004 \\
\hline $\begin{array}{l}\text { Complement C1q subcomponent } \\
\text { subunit A }\end{array}$ & 0.83 & 0.001 & 0.78 & $<0.001$ & 0.70 & $<0.001$ \\
\hline Coagulation factor $V$ & 0.75 & 0.019 & 0.72 & 0.006 & 0.68 & 0.004 \\
\hline Vitamin D-binding protein & 0.81 & 0.002 & 0.69 & $<0.001$ & 0.67 & $<0.001$ \\
\hline Coagulation factor XII & 0.72 & 0.022 & 0.63 & 0.002 & 0.54 & 0.001 \\
\hline Apolipoprotein M & 0.83 & $<0.001$ & 0.60 & $<0.001$ & 0.50 & $<0.001$ \\
\hline Platelet factor 4 variant & 0.59 & 0.011 & 0.42 & 0.001 & 0.35 & $<0.001$ \\
\hline Haptoglobin & 8.44 & $<0.001$ & 1.38 & 0.033 & 1.24 & 0.080 \\
\hline S100A9 & 1.37 & 0.085 & 1.68 & 0.006 & 2.22 & $<0.001$ \\
\hline
\end{tabular}

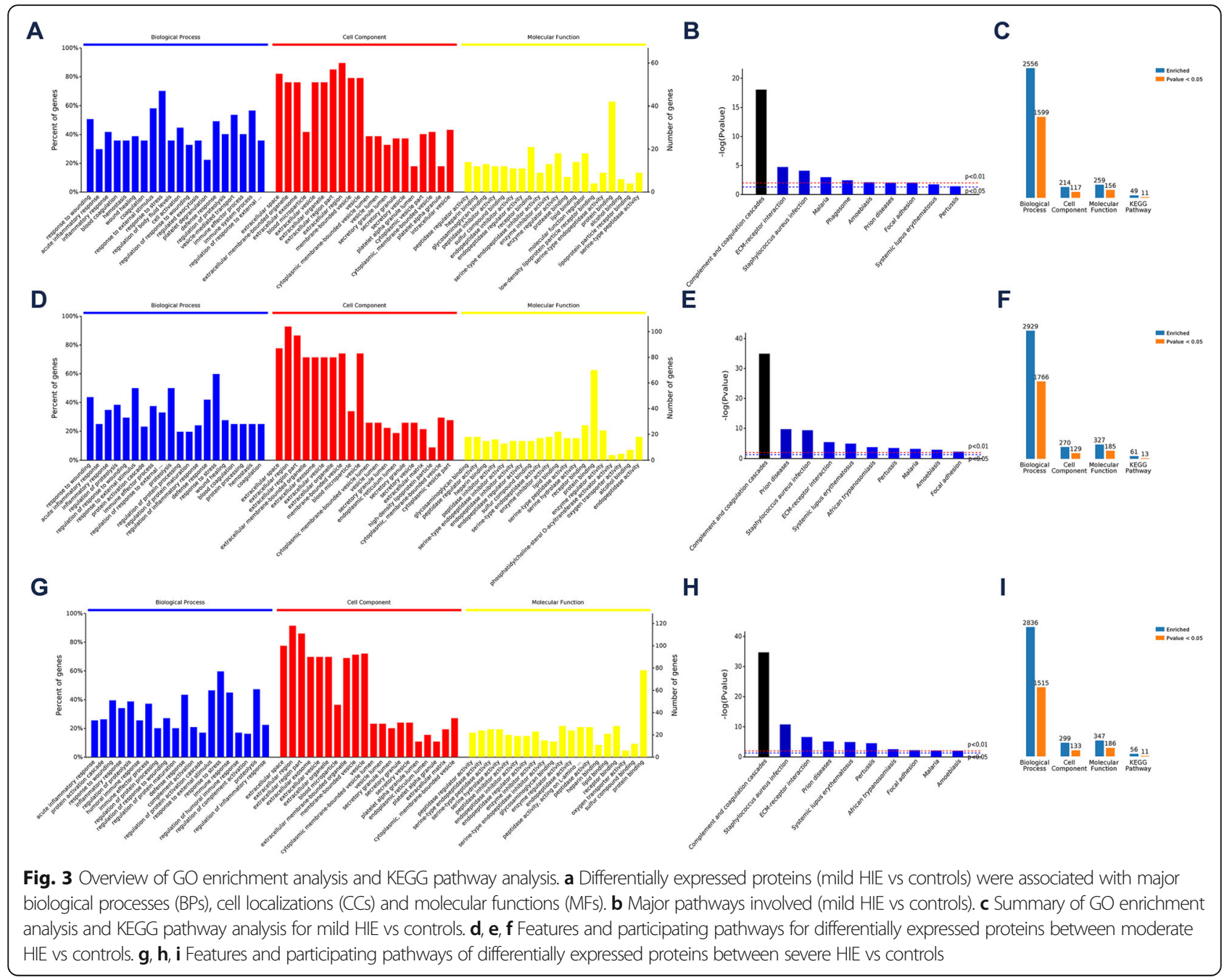




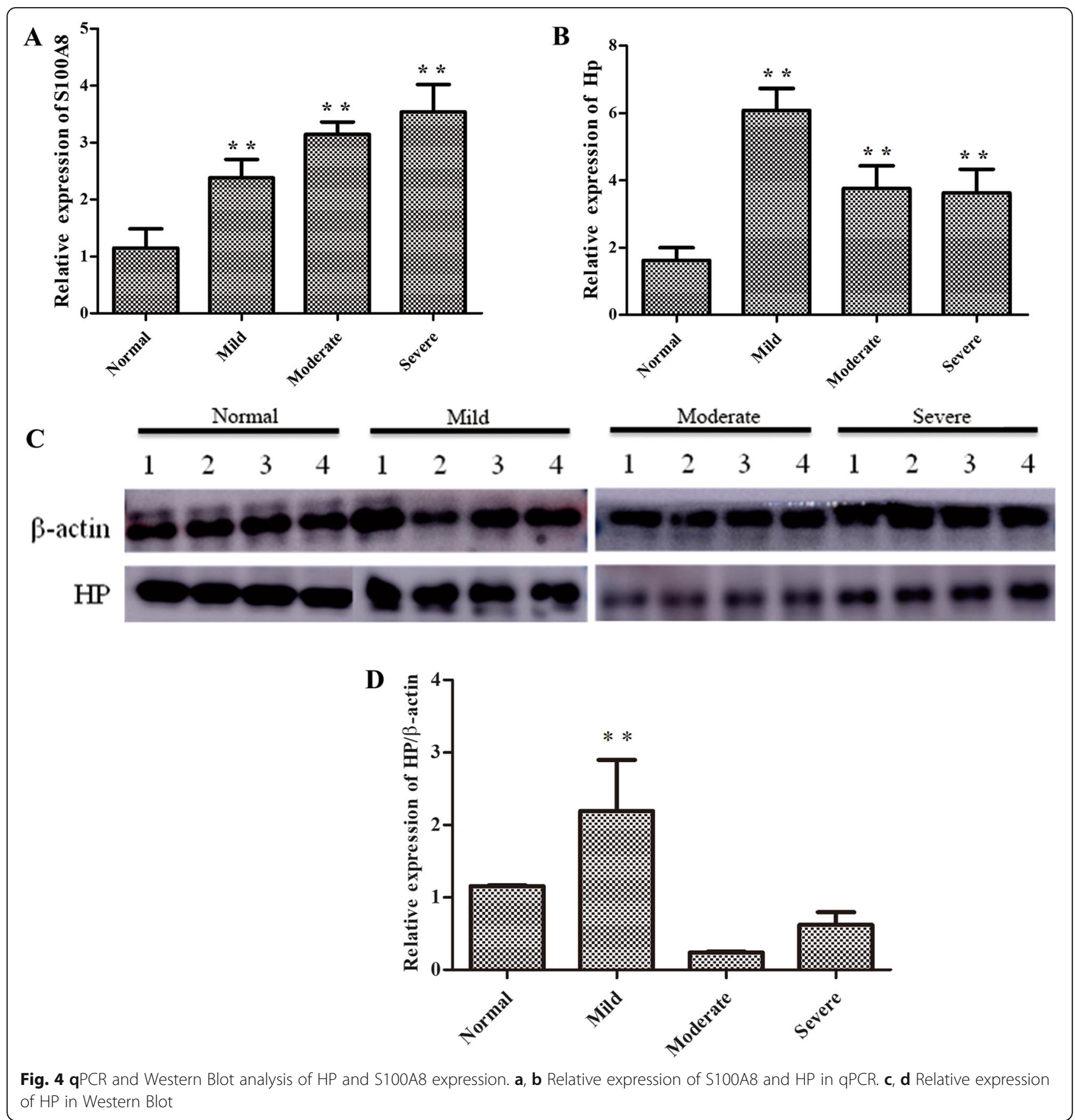

[4]. Predictive or prognostic biomarkers that could guide therapeutic strategies may be of critical importance.

\section{Conclusions}

Our study based on iTRAQ identified S100A8 and HP protein as a potential biomarker for neonatal HIE and the complement and coagulation cascades as important in the development of neonatal HIE.

\section{Abbreviations}

HIE: Neonatal hypoxic ischemic encephalopathy; NSE: Neuron-specific enolase IL-6: Interleukin-6; iTRAQ: Isobaric tag for absolute and relative quantitation;
IgG: Immunoglobulin; SDS: Sodium dodecyl sulfate; PAGE: Polyacrylamide gel electrophoresis; ACN-H2O-FA: Acetonitrile/water/formic acid; AGC: Automatic gain control; IT: Ion accumulation time; NCE: Normalized collision energy; MGF: Mascot generic format; FDR: False discovery rate; KEGG: Kyoto Encyclopedia of Genes and Genomes; GO: Gene ontology; PCR: Polymerase chain reaction; HP: Haptoglobin; APOE: Apolipoprotein E; APOM: Apolipoprotein M; PVDF: Polyvinylidene fluoride

\section{Acknowledgements}

Not applicable.

Authors' contributions

$X L X$ and $Z Y Y$ were primarily responsible for study design, data analysis and manuscript preparation. YYJ participated in the sample collection and 
prepared the draft manuscript. JMF and LG performed western blotting and qPCR experiments. LH and FX were involved in the clinical data analysis. SB contributed to the study design and provided valuable suggestions for conducting the experiments. All authors read and approved the final manuscript.

\section{Funding}

This work was supported by the National Natural Science Foundation of China (No. 81471488, 81671532, 81771625, 81871193); Jiangsu Provincial Key Medical Discipline (No. ZDXKA2016013); the Jiangsu Province Key Research and Development of Special Funds in China (No. BE2015644); Jiangsu Provincial Medical Youth Talentthe Jiangsu Provincial Medical Youth Talent (QNRC2016758, QNRC2016763); the Jiangsu Province Women and Children Health Research Project (No. F201750); the Public Health Technology Project of Suzhou City of China (SYS201765); the Department of Pediatrics Clinical Center of Suzhou City of China (Szzx201504).

\section{Availability of data and materials}

The datasets used and/or analyzed during the current study are available from the corresponding author on reasonable request.

\section{Ethics approval and consent to participate}

This study was approved by the Hospital Ethics Committee (Ethics No. 2015LW002), and informed consent was obtained from the patients' legal guardians.

\section{Consent for publication}

Not applicable.

\section{Competing interests}

The authors declare no conflicts of interest.

Received: 27 September 2019 Accepted: 27 April 2020 Published online: 24 May 2020

\section{References}

1. Buonocore G, Perrone $S$, Longini $M$, et al. Non protein bound iron as early predictive marker of neonatal brain damage. Brain. 2003;126:1224-30.

2. Shao XM, Ye H, Qiu XS. Practical newborn[M]. 4th ed. Beijing: People's Medical Publishing House; 2011. p. 395

3. Yang L, Li D, Chen S. Hydrogen water reduces NSE, IL-6, and TNF-alpha levels in hypoxic-ischemic encephalopathy. Open Med (Wars). 2016;11:399406.

4. Massaro AN, Wu YW, Bammler TK, et al. Plasma biomarkers of brain injury in neonatal hypoxic-ischemic encephalopathy. J Pediatr. 2018;194:67-75.e1.

5. Graham EM, Everett AD, Delpech JC, et al. Blood biomarkers for evaluation of perinatal encephalopathy: state of the art. Curr Opin Pediatr. 2018;30: 199-203.

6. Douglas-Escobar M, Weiss MD. Biomarkers of hypoxic-ischemic encephalopathy in newborns. Front Neurol. 2012;3:144.

7. Monti C, Zilocchi M, Colugnat I, et al. Proteomics turns functional. J Proteome. 2019;198:36-44.

8. Group of Neonatology, Chinese Pediatric Society, Chinese Medical Association. Diagnostic criteria for neonatal hypoxic-ischemic encephalopathy. Zhonghua Er Ke Za Zhi. 2005;43:584.

9. Viemann D, Strey A, Janning A, et al. Myeloid-related proteins 8 and 14 induce a specific inflammatory response in human microvascular endothelial cells. Blood. 2005;105:2955-62.

10. Ehrchen JM, Sunderkotter C, Foell D, et al. The endogenous toll-like receptor 4 agonist S100A8/S100A9 (calprotectin) as innate amplifier of infection, autoimmunity, and cancer. J Leukoc Biol. 2009;86:557-66.

11. Sunahori K, Yamamura M, Yamana J, et al. The S100A8/A9 heterodimer amplifies proinflammatory cytokine production by macrophages via activation of nuclear factor kappa B and p38 mitogen-activated protein kinase in rheumatoid arthritis. Arthritis Res Ther. 2006;8:R69.

12. Halayko AJ, Ghavami S. S100A8/A9: a mediator of severe asthma pathogenesis and morbidity? Can J Physiol Pharmacol. 2009;87:743-55.

13. Nordal HH, Brokstad KA, Solheim M, et al. Calprotectin (S100A8/A9) has the strongest association with ultrasound-detected synovitis and predicts response to biologic treatment: results from a longitudinal study of patients with established rheumatoid arthritis. Arthritis Res Ther. 2017;19:3.
14. Bianchi ME, Manfredi AA. Immunology. Dangers in and out. Science. 2009; 323:1683-4.

15. Ma L, Sun P, Zhang JC, et al. Proinflammatory effects of S100A8/A9 via TLR4 and RAGE signaling pathways in BV-2 microglial cells. Int J Mol Med. 2017; 40:31-8.

16. Sadrzadeh SM, Bozorgmehr J. Haptoglobin phenotypes in health and disorders. Am J Clin Pathol. 2004;121(Suppl):S97-104.

17. Glushakov AV, Arias RA, Tolosano E, et al. Age-dependent effects of Haptoglobin deletion in neurobehavioral and anatomical outcomes following traumatic brain injury. Front Mol Biosci. 2016;3:34.

18. Zhao X, Song S, Sun G, et al. Neuroprotective role of haptoglobin after intracerebral hemorrhage. J Neurosci. 2009;29:15819-27.

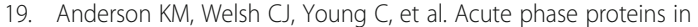
cerebrospinal fluid from dogs with naturally-occurring spinal cord injury. J Neurotrauma. 2015;32:1658-65.

20. Zhao X, Song S, Sun G, et al. Cytoprotective role of haptoglobin in brain after experimental intracerebral hemorrhage. Acta Neurochir Suppl. 2011; 111:107-12.

21. Melis JP, Strumane K, Ruuls SR, et al. Complement in therapy and disease: regulating the complement system with antibody-based therapeutics. Mol Immunol. 2015;67:117-30.

22. Yang S, Nakamura T, Hua Y, et al. The role of complement C3 in intracerebral hemorrhage-induced brain injury. J Cereb Blood Flow Metab. 2006;26:1490-5.

23. Alexander JJ. Blood-brain barrier (BBB) and the complement landscape. Mol Immunol. 2018;102:26-31.

24. Shah TA, Nejad JE, Pallera HK, et al. Therapeutic hypothermia modulates complement factor $\mathrm{C} 3 \mathrm{a}$ and $\mathrm{C} 5 \mathrm{a}$ levels in a rat model of hypoxic ischemic encephalopathy. Pediatr Res. 2017;81:654-62.

25. Bauman ME, Cheung PY, Massicotte MP. Hemostasis and platelet dysfunction in asphyxiated neonates. J Pediatr. 2011;158:e35-9.

26. Shah P, Riphagen S, Beyene J, et al. Multiorgan dysfunction in infants with post-asphyxial hypoxic-ischaemic encephalopathy. Arch Dis Child Fetal Neonatal Ed. 2004;89:F152-5.

27. Castle V, Andrew M, Kelton J, et al. Frequency and mechanism of neonatal thrombocytopenia. J Pediatr. 1986;108:749-55.

28. Roberts IA, Murray NA. Thrombocytopenia in the newborn. Curr Opin Pediatr. 2003;15:17-23.

29. Suzuki S, Morishita S. Hypercoagulability and DIC in high-risk infants. Semin Thromb Hemost. 1998;24:463-6.

30. Buchanan GR. Coagulation disorders in the neonate. Pediatr Clin N Am. 1986:33:203-20.

31. Wolberg AS, Meng ZH, Monroe DM 3rd, et al. A systematic evaluation of the effect of temperature on coagulation enzyme activity and platelet function. J Trauma. 2004;56:1221-8.

32. Rohrer MJ, Natale AM. Effect of hypothermia on the coagulation cascade. Crit Care Med. 1992;20:1402-5.

\section{Publisher's Note}

Springer Nature remains neutral with regard to jurisdictional claims in published maps and institutional affiliations.

Ready to submit your research? Choose BMC and benefit from:

- fast, convenient online submission

- thorough peer review by experienced researchers in your field

- rapid publication on acceptance

- support for research data, including large and complex data types

- gold Open Access which fosters wider collaboration and increased citations

- maximum visibility for your research: over $100 \mathrm{M}$ website views per year

At $\mathrm{BMC}$, research is always in progress.

Learn more biomedcentral.com/submissions 